Hollande, des dons pour l'œuvre de la Croix-Rouge au Portugal.

Cette dernière a vu, en effet, son travail s'étendre par l'envoi du corps expéditionnaire en France pour comhattre à côté des soldats anglais et français. Le Comité central de Lisbonne a dû préparer l'envoi d'un détachement sanitaire important, comprenant des médecins, des infirmiers et des infirmières, au total de 80 personnes. Cette ambulance était destinée à l'hôpital portugais établi à Boulogne-sur-Mer; il occupe un bâtiment spécial que la Croix-Rouge anglaise faisait construire. Cet hôpital comprend 2 à 300 lits et ses frais d'entretien dépassent les ressources de la Croix-Rouge portugaise, qui fait appel à l'appui des pays neutres.

Il vaut la peine de relever la solidarité internationale qui se manifeste ainsi, trois pays se trouvant intéressés à cette initiative, la Hollande où le Comité nouveau s'est fondé, la Grande-Bretagne qui prête son concours pour les constructions, le Portugal enfin qui est le bénéficiaire de l'entreprise.

Il s'agit là encore, il est vrai, d'une de ces nouvelles sections étrangères, constituées sur territoire national, dont nous avons été appelés à parler plus d'une fois ${ }^{1}$, mais nous ne doutons pas que la Croix-Rouge portugaise ne se soit préalablement mise pleinement d'accord avec la Croix-Rouge néerlandaise et n'ait obtenu son agrément à la constitution et à l'action de ce nouveau Comité sur territoire hollandais.

\title{
RUSSIE
}

\section{Les effets de la révolution musse sur la Croix-Rouge}

(Deuxième article)

Les excellents rapports qui ont toujours existé entre le Comité International de la Croix-Rouge et la Direction

1 Voy. T. XLVIII, 1917, p. 422. 
générale de la Croix-Rouge russe nous font suivre avec un douloureux intérêt la crise dangereuse qui menace de compromettre irrémédiablement l'existence de cette Société qui, depuis sa fondation, a donné tant de preuves d'activité et de dévouement. L'ancienne direction, dont nous avons connu personnellement les membres et dont nous avons pu apprécier la haute valeur, a presque entièrement disparu. Il était impossible que le régime de désordre et d'incohérence qui sabote actuellement toutes les institutions de la Russie, épargnât la Croix-Rouge.

Dans notre dernière chronique (octobre 1917) ${ }^{1}$, nous avons exposé, d'après les documents officiels, les procédés mis en ceuvre pour "démocratiser" la Croix-Rouge russe. Nous avons cité la Déclaration par laquelle la Première Conférence nationale des Travailleurs de la Croix-Rouge, qui s'était adjugé le rôle d'une constituante omnipotente, accusait la Direction générale d'être réactionnaire, antidémocratique et incapable de gérer les affaires d'une CroixRouge renouvelée et libérée. La Direction générale répondit à cet acte d'accusation par un exposé digne et modéré de son activité passée. Son Vice-Président, M. Pokrovsky, se rendit auprès des délégués de la Conférence, pour les inviter à venir discuter les termes de cette déclaration avec les membres de la Direction. Mais la Conférence, dảns sa séance du 9 juillet 1917, se refusa à cette discussion et maintint les termes de sa déclaration.

Ce qu'il y a de très intéressant à noter, c'est que le Gouvernement Provisoire appuie nettement la Direction générale. Son Commissaire spécial, M. P. A. Velitchkof, s'adressant à la Conférence, s'est exprimé de la manière suivante : "J'ignore à quel point cette Conférence peut se déclarer munie de pleins pouvoirs et de quelle manière elle entend réorganiser la Croix-Rouge; je sais seulement que le Gouvernement provisoire et la Direction générale considèrent cette assemblée comme une réunion préparatoire en vue du Congrès national qui doit se réunir prochainement.

\footnotetext{
1 Voy. T. XIVIII, 1917, p. 458.
} 
La tâche de la Conférence doit se borner à centraliser les voux des assemblées partielles tenues sur tous les fronts et dans tous les rayons. Sa Déclaration, que j'appelle un beau morceau littéraire, renferme à l'égard de la Direction générale des accusations offensantes et injustifiées. Elle a profondément blessé les anciens et dévoués collaborateurs de la Groix-Rouge. En ce qui me concerne, je proclame que l'ancienne Direction générale a bien rempli les nombreux devoirs qui lui incombaient, et c'est comme témoin, comme officier du front que j'ai acquis cette conviction."

M. Velitchkof, avec un courage que l'hostilité de l'assemblée rendait méritoire, démontra que ce serait une grave erreur que de se priver des services d'un personnel expérimenté pour remettre l'œuvre entre les mains de nouveaux venus, nullement préparés à une tâche aussi importante que difficile.

Mais ces considérations ne pouvaient être d'aucun poids auprès d'une assemblée dont le siège était fait d'avance. L'épuration de la Direction générale, pratiquée en avril, ne paraissait plus suffisante. Comme toujours dans les périodes révolutionnaires, les éléments avancés de la veille sont les réactionnaires du lendemain. Il fallait introduire dans la Direction générale un élément plus démocratique que le précédent. La Conférence décida de nommer vingt nouveaux membres de la Direction générale. Dans la liste élaborée et adoptée, nous trouvons 1 professeur, 6 docteurs, 6 infirmiers, 2 sœurs de charité, 2 chauffeurs, 6 ex-soldats, décorés maintenant du titre d'employés militaires, etc.

Dans la séance du 13 juillet, le Commissaire du Gouvernement provisoire, M. Vélitchkof, s'opposa à la nomination d'un contingent aussi nombreux et déclara que le Gouvernement ne sanctionnerait que l'élection de 10 nouveaux membres, "en attendant que la situation politique futt plus éclaircie et plus déterminée. "

Il fit remarquer que la décision de la Conférence d'attribuer un traitement pécuniaire aux membres de la Direction générale contrastait avec l'ancien régime qui n'admettait pas de rétribution pour ces fonctions. 
Sur ce dernier point l'assemblée maintint sa résolution, qu'elle motiva en déclarant «qu'avant la révolution les titulaires des fonctions gratuites étaient. des incapables qui favorisaient la dilapidation des deniers nationaux. "

Après le départ de M. Vélitchkof, la Conférence discuta encore le nombre des candidats à nommer et se décida enfin à le réduire provisoirement à 10, "considérant que le Gouvernement provisoire et les Soviets sont trop occupés, pendant la crise actuelle, pour avoir le temps d'entrer en relations avec tous les délégués de la Conférence. "Une nouvelle sélection fut donc opérée et donna comme résultat la nomination des 10 nouveaux membres de la Direction générale, à savoir 1 professeur, 3 docteurs, 2 infirmiers et 4 "employés militaires". Nous croyons inutile de citer les noms.

Pour consoler les candidats évincés, la Conférence vota à l'unanimité la résolution suivante :

"1. La Conférence interrompt ses travaux, mais ne se " dissout pas et peut être convoquée en tout temps par "le Comité Exécutif.

" 20 Les 20 candidats désignés par la Conférence forment "le Comité Exécutif.

« 3० Les 10 candidats élus comme membres de la Direc" tion générale de la Croix-Rouge forment le Bureau du "Comité Exécutif.

" $4^{\circ}$ Ils s'engagent à faire entrer le plus tôt possible les * 10 autres candidats dans la Direction générale.

" $5^{\circ}$ Le Comité Exécutif est chargé de la publication "des travaux de la Conférence. "

La fin de la séance fut consacrée à des considérations politiques et à des félicitations mutuelles sur la "démocratisation " de la Direction générale.

Un incident faillit jeter une ombre sur la satisfaction éprouvée par la Conférence. Une assemblée, tenue par les employés de la Chancellerie de la Direction générale et des institutions de la Croix-Rouge, s'était nettement déclarée opposée aux décisions de la Conférence et avait envoyé une 
protestation formelle. Ce document constatait que la Conférence, convoquée uniquement poủr jeter les bases et préparer les matériaux du Congrès national de la Croix-Rouge, avait dépassé les bornes de ses compétences; que les élections, faites dans un but déterminé, ne donnaient aux délégués aucun mandat de se transformér en Constituante; que ces élections d'ailleurs avaient été faites irrégulièrement et que par conséquent les résolutions de la Conférence n'avaiont aucune valeur légale. L'assemblée protestait contre les accusations tendancieuses formulées contre la CroixRouge russe et réfutées par toute I'histoire de.celle-ci, particulièrement pendant la guerre présente, et contre la complète incompétence des délégués, à la Conférence qui ne connajssaient ni la situation internationale de la CroixRouge, ni les conditions de son activité, ni les nécessités spéciales de l'époque actuelle. Les promésses faites par la Conférence constituaient simplement un procédé démagogique de la part de gens qui, n'ayant avec la Croix-Rouge aucune attache permanente, ne pourraient tenir aucun des engagements qu'ils prenajent. L'assemblée s'êlevait contre l'introduction de 20 nouveaux membres dans la Direction générale, mesure aussi illégale que dangereuse pour l'œuvre de la Croix-Rouge, et protestait énergiquement contre toutes les tentatives faites par la Conférence pour mener à la ruine l'institution de la Croix-Rouge, et cela dans une épcque aussi grave pour la patrie.

Cette protestation fut présentée au Président de la Douma, au Président par intérim de la Direction générale de la Croix-Rouge, au Commissaire du Gouvernement provisoire et au représentant du ministère de la Guerre.

La Conférence se trouvait donc en présence d'une opposition formelle, venue d'un milieu identique au sien, celui des employés des institutions de la Croix-Rouge. Mais elle ne s'en émut guère et se borna à voter une résolution de plus : "La Première Conférence nationale des travailleurs de la "Croix-Rouge considère comme au-dessous de sa dignité "d'entrer en discussion sur la protestation des soutiens "de l'ancien régime." 
Sur cette dédaigneuse réponse, la Conférence déclara sa session close.

La nomination des 10 nouveaux membres de la Direction générale mettait fin au mandat provisoire des délégués des Soviets, nommés en avril ${ }^{1}$.

La première séance de la Direction générale fut consacrée à mettre en contact les nouveaux élus avec l'ancien personnel. On échangea des compliments oratoires. Au nom des 10 élus, le Dr Gorasch promit de consacrer à l'œuvre de secours aux blessés toutes leurs forces, leurs talents et leur expérience. Conformément à la technologie révolutionnaire en Russie, dès la séance suivante, le 19 juillet, le nouveau groupe proposait la formation de ncmbreux comités : 10 Conseil médico-sanitaire; $2^{\circ}$ Comité de la presse et de la propagande des idées de la Croix-Rouge; $3^{\circ}$ Comité des besoins professionnels des travailleurs de la Croix-Rouge, avec un Sous-Comité de l'instruction et de la culture; t $^{\mathrm{O}}$ Commission pour la réunion du Congrès général de la CroixRouge; $5^{\circ}$ Bureau de la Direction générale; en outre, Commissions spéciales pour les secours aux travailleurs de la Croix-Rouge, pour les récompenses et subsides, pour les bibliothèques, pour les lectures et conférences, pour la préparation 'du personnel, etc., etc.

Cette inépuisable liste de comités et de commissions paraissait à ces théoriciens la condition indispensable et le gage infaillible d'une vaste et utile àctivité. Malgré un peu d'opposition, cette réorganisation fut adoptée.

Pendant qu'à Pétrograde l'incompétence et l'irresponsabilité envahissaient ainsi les services de la Croix-Rouge, que se passait-il sur le front?

Tant que le personnel qui travaillait dans la zone des opérations de guerre se sentit soutenu et dirigé par l'autorité centrale, il remplit sa tâche avec conscience et avec dévoucment dans des circonstances devenant de jour en jour plus difficiles. Un ordre du jour, en date du 21 juillet, du général

1 Voir T. XLVIII, 1917, p. 459. 
Kornilof, alors commandant en chef du front S.-O., rend justice à la vaillante conduite des colonnes de la CroixRouge pendant la retraite de Galicie et de Bukovine. "Je constate avec un sentiment de profonde satisfaction que les services de la Croix-Rouge ont, sous le feu de l'ennemi et malgré de lourdes pertes en personnel, accompli leur tâche sacrée jusqu'à la limite du possible, en dépit de la panique et de la désorganisation qui ont marqué la retraite des troupes. Ce travail dévoué prouve les brillantes qualités du personnel, sa fermeté, son sentiment du devoir et son abnégation. Je suis fier d'exprimer à tout le personnel de la CroixRouge ma profonde reconnaissance et celle de l'armée toute entière. "Cet ordre du jour rend un hommage particulier au Délégué général de la Croix-Rouge, sénateur Ivanitzky pour avoir, "dans cette période pénible où l'armée se décomposait et s'éparpillait, maintenu dans les services de la CroixRouge la discipline et l'esprit de dévouement aux souffrances des blessés ».

Mais lorsque les chefs eux-mêmes sont abandonnés par l'autorité centrale, leurs efforts pour maintenir l'ordre sont condamnés à l'impuissance. Les soldats quittant leurs postes, les infirmiers en font autant. Les sœurs de charité désertent en masse. Tandis que depuîs le début de la guerre elles s'étaient présentées par milliers pour servir sous le drapeau de la Croix-Rouge, elles lâchent maintenant en hâte les hôpitaux et leurs malades, sans paraître se soucier des conséquences de cet exode. Les médecins restent sans personnel sanitaire. En vain, le Congrès des sceurs de charité du front nord adresse-t-il un appel désespéré au nom de l'intérêt de la patrie et du sentiment de la discipline. La désertion continue.

Ce serait faire tort aux sentiments des femmes russes qui, pour la plupart, avaient considéré le service des malades et des blessés comme un apostolat sacré et qui ont supporté les dangers et les misères de trois ans de guerre, que de constater cet abandon de leurs fonctions sans en donner quelques justification.

Le personnel choisi et soigneusement préparé, qui avait 
été fourñi au début par les Communautés de sours de chärité, était rapidement devenu insuffisant. On avait da recrutér à la hâte toutes lés bonnes volontés qui se présentaient. Une préparation théorique et pratique de quelques semaines suffisait pour faire admettre les nouvelles recrues, mais non pour les rendre aptes au service pénible et exigeant qu'on attendait d'elles. Il y eut beaucoup de déchets. Le premier Congrès des médecins des armées se plạignit fortement des nombreux cas d'indiseipline et même d'inconduite survenus dans leurs services. Les sœurs de charité furent moins considérées, moins bien traitées. A mesure que la guerré se prolongeait, le niveau moral et intellectuel des sœurs fléchissait. Un antagonisme regrettable s'était créé entre elles et les sanitaires, dont la valeur morale avait aussi baissé. Leurs querelles entravaient le service.

Chose; curieuse, tandis que les blessés qui passent dans les lazarets un temps relativement court, témoignent d'une façon touchante leur reconnaigsance enveps les soours de charité, les malades séjournant longtemps dans les hopitaux subissent l'influence des infirmiers et traitent les sceurs avec une grossièreté et une hostilité révoltantes. Dès que leur état de santé s'améliore quelque peu, les malades sont saisis de la fièvre des meetings. On élit des comités ehargés de surveiller le traitement, la nourriture et le régime de l'hôpital. La Direction générale est saisie de pétitions réclamant le droit pour les députés des malades de faire partie des conseils médicaux et économiques des Communautés de sœurs de charité. On nous a affirmé qu'en France, dans des hôpitaux où étaient traités des blessés russes, cette même tendance d l'insubordination organisée s'était mañifestée à maintes reprises.

En outre, par suite du renchérissement énorme de tons les produits de consommation, le salaire des sceurs de charité, fixé à 40 roubles par mois, est devenu absolument insuffisant. Il n'y a donc rién de surprenant si, dans le désarroi général, ces pauvres femmes réduites aux privations, n'étant plus soutenues ni par les" autorités supérieures ni par le sentiment de l'utilité de leurs efforts, abandonnent une tâche impossible à remplir et retournent dans leurs foyers. 
Cette situation critique a decidé la Direction générale a porter le salaire des scurs de charite de 40 a 70 roubles par mois, augmentation qui correspond à un surcroît de depense annuelle de 12 millions de roubles.

La situation militaire et politique de la Russie s'est si complètement modifiêe dans les derniers mois de 1917 , que les renseignements que nous venons de donner n'orit plus qu'un intérêt historiquè. Quel est actuèllement l'état de la Croix-Rouge russe? Faute de renseignements précis, on doit se borner aux conjectures les plus probables.

La suspension des opérations de guerre n'implique pas un arrêt immédiat de l'activité de la Croix-Rouge. Il reste encore des hôpitaux pleins de malades et de blessés. Il faut secourir et ravitailler les troupes évacuées dans des conditions exceptionnellement difficiles. Il faut organiser avec prudence le licenciement du personnel et le retrait du matériel, pour eviter des pertes énormes. Selon toute probabilité, les luttes intérieures se prolongeront longtemps encore, et ta Croix-Rouge doit se préparer à une nouvelle tâche, d'autant plus terrible qu'elle sera la conséquence de la guerre civile.

L'emiettement politique du pays va soustraire à l'autorité de la Direction genérale me quantifé considérable d'institutions provinciales, d'ceuvres et de ressources sur lesquelles etle pouvait compter jusqu'a présent. I parait presque certain que les portions de la Russie qui aspirent a l'autonomie par le séparatisme appliqueront ce principe à toutés leurs institutions $y$ compris la Croix-Rouge:

Nous verrons se créer, une Croix-Rouge finlandaise, une Croix-Rouge de l'Ukraine, une Croix-Rouge du Turkestan ou du Caucase Ce fractionnement presque inévitable de la Groix-Rouge russe amènera des difficultés d'ordre général qui peuvent, à juste titre, préoccuper le Comité International.

Pour le moment, la tâche de la Croix-Rouge russe est 1 a plus ardue qu'il soit possible d'imaginer. Les subsides du Gouvernement doivent forcément tomber a un niveau très bas. Les contributions volontaires des particuliers diminuent, aussi bien par le fait de l'appauvrissement géné- 
ral que par suite de la désaffection pour une institution naguère si populaire. Il est vrai qu'en contrepartie les dépenses dites de guerre subiront une réduction notable. Ce n'est pas la situation financière, si grave qu'elle sòit, qui peut mettre en péril l'existence de la Croix-Rouge russe. C'est surtout au rétablissement de l'ordre dans les services, de l'unité dans la direction, de la discipline à tous les degrés du personnel, que doit tendre l'activité de ceux qui se sont placés eux-mêmes à la tête de la Croix-Rouge russe. Leur responsabilité est lourde. L'histoire les jugera, non sur leurs théories, mais sur les résultats de leur action.

F. THORMEYER.

\section{SAXE}

\section{Décès du Président de la Croix-Rouge saxonne} S. E. D.-Otto comte Vitzthum von Ecketidt

Au moment de livrer à l'impression le présent numéro, nous apprenons avec regret le décès, survenu le 17 décembre 1917, du vénéré président de la Croix-Rouge saxonne, S. E. D. Otto comte Vitzthum von Eckstädt.

Tout en adressant à la Société qui perd son chef l'expression de nos sincères compliments de condoléance, nous devons nous borner, pour le moment, à annoncer ce déçès, comptant sur le Comité de Dresde pour nous fournir, en vue de notre prochain numéro, un article nécrologique avec portrait.

\section{SUĖDE}

\section{Le Doctorat honoris causa conféré au prince Charles de Suede par I'Université d'Upsal}

M. A. Mohn, de la Croix-Rouge suédoise, nous envoie irticle suivant, et nous sommes heureux de pouvoir nous 